\title{
Figures and Charts
}

Sigurður Ingibergur Björnsson, Steingrímur Páll Kárason, Jón Karl Helgason Stylometry and the Faded Fingerprints of Saga Authors

Charts 1-9: Created by Steingrímur Páll Kárason.

Lena Rohrbach

The Persistence of the Humanistic Legacy

Fig. 1: Copenhagen, Arnamagnæan Collection, AM 47 fol., f. 1r. Photo: Suzanne Reitz. Used with permission.

Fig. 2: Copenhagen, Arnamagnæan Collection, AM 45 fol., f. 1v. Photo: Suzanne Reitz. Used with permission.

Fig. 3: Copenhagen, Arnamagnæan Collection, AM 45 fol., f. 1r. Photo: Suzanne Reitz. Used with permission.

Fig. 4: Copenhagen, Arnamagnæan Collection, AM 47 fol., f. 72v. Photo: Suzanne Reitz. Used with permission.

Fig. 5: Copenhagen, Arnamagnæan Collection, AM 47 fol., f. 129r, detail. Photo: Suzanne Reitz. Used with permission.

Fig. 6: Copenhagen, Arnamagnæan Collection, AM 47 fol., f. 139v, detail. Photo: Suzanne Reitz. Used with permission.

Fig. 7: Copenhagen, Arnamagnæan Collection, AM 47 fol. f. 1v. Photo: Suzanne Reitz. Used with permission.

Fig. 8: Copenhagen, Arnamagnæan Collection, AM 47 fol., f. 10r. Photo: Suzanne Reitz. Used with permission.

Fig. 9: Copenhagen, Arnamagnæan Collection, AM 45 fol., f. 37r. Photo: Suzanne Reitz. Used with permission.

Fig. 10: Copenhagen, Arnamagnæan Collection, AM 45 fol,, f. 84r. Photo: Suzanne Reitz. Used with permission.

Fig. 11: Snorre Sturlusøns Norske Kongers Chronica, udsat paa Danske aff H. Peder Claussøn, Copenhagen 1633. Title page. Public Domain. https://baekur.is/bok/000365900/Snorre_Sturlessons_ Norske.

Fig. 12: Snorre Sturlusøns Norske Kongers Chronica, udsat paa Danske aff H. Peder Claussøn, Copenhagen 1633, p. 320-321. Public Domain. https://baekur.is/bok/000365900/Snorre_Sturlessons_ Norske.

Fig. 13: Snorre Sturlusøns Norske Kongers Chronica, udsat paa Danske aff H. Peder Claussøn, Copenhagen 1633, p. 500-501. Public Domain. https://baekur.is/bok/000365900/Snorre_Sturlessons_ Norske.

Fig. 14: Snorre Sturlusøns Norske Kongers Chronica, udsat paa Danske aff H. Peder Claussøn, Copenhagen 1633, p. 795. Public Domain. https://baekur.is/bok/000365900/Snorre_Sturlessons_ Norske. 
Fig. 15: Snorre Sturlusøns Norske Kongers Chronica, udsat paa Danske aff H. Peder Claussøn, Copenhagen 1633, p. 796-797. Public Domain. https://baekur.is/bok/000365900/Snorre_Sturlessons_ Norske.

Fig. 16: Heimskringla eller Snorre Sturlusons Nordländske Konunga Sagor, ed. by Johan Peringskiöld, Stockholm 1697. Public Domain. https://baekur.is/bok/000365879/Heimskringla.

Fig. 17: Copenhagen, Arnamagnæan Collection, AM 435 a 4to, f. 37v. Photo: Suzanne Reitz. Used with permission.

Fig. 18: Copenhagen, Arnamagnæan Collection, AM 435 b 4to, f. 1r. Photo: Suzanne Reitz. Used with permission.

Fig. 19: Copenhagen, Arnamagnæan Collection, AM 456 fol, f. 2v/3r. Photo: Suzanne Reitz. Used with permission.

Gudrun Bamberger

A Theory of Early Modern Authorship

Fig. 1: Historia von D. Johann Fausten. Frankfurt am Main: Johann Spies 1587, Titel [VD16 F 943]. Herzog August Bibliothek Wolfenbüttel, Sig. A: 56.3 Eth. 